\title{
Status of direct neutrino mass measurements
}

\section{Florian Fränkle*}

Karlsruhe Institute of Technology

E-mail: florian.fraenkleakit.edu

\begin{abstract}
The absolute neutrino mass scale is one of the big open questions in particle physics, astrophysics and cosmology. Cosmological observations and neutrinoless double beta decay experiments provide an indirect access to the absolute neutrino mass scale, but are model-dependent. A model independent, direct approach to determine the neutrino mass is the precise investigation of weak decays. The KATRIN experiment, which is currently under construction, will improve the neutrino mass sensitivity to $200 \mathrm{meV} / \mathrm{c}^{2}$ via the precise measurement of the tritium $\beta$-decay spectrum close to the endpoint energy with a high-resolution electrostatic spectrometer. Experiments using alternative approaches such as spectroscopy via coherent cyclotron radiation or calorimetric measurements of ${ }^{163} \mathrm{Ho}$ electron capture are being developed. This article briefly reviews the current status of different direct neutrino mass measurement experiments.
\end{abstract}

XIIth International Conference on Heavy Quarks \& Leptons 2014

25-29 August 2014

Schloss Waldthausen, Mainz, Germany

${ }^{*}$ Speaker. 


\section{Introduction}

The current understanding of neutrinos is that neutrino flavor eigenstates are connected to neutrino mass eigenstates via the lepton mixing matrix ${ }^{1} U$. A neutrino flavor eigenstate is therefore a superposition of different mass eigenstates. Various experiments with atmospheric, solar, accelerator and reactor neutrinos showed that neutrinos oscillate from one flavor state into another during flight, which implies that neutrinos are massive particles. With the help of these oscillation experiments the mixing matrix $U$ containing the mixing angles as well as the differences between the squares of the neutrino mass eigenstates can be determined [1]. However, neutrino oscillations are not sensitive on the absolute neutrino mass scale and therefore can only provide a lower limit.

The knowledge of neutrino masses is very important for astrophysics and cosmology in order to describe the role of neutrinos in the evolution of the universe. There are 336 neutrinos $/ \mathrm{cm}^{3}$ left over from the Big Bang, which makes neutrinos one of the most abundant particles in the universe (about $10^{9}$ times more abundant than atoms). Even with a small mass, neutrinos contribute to the energy density of the universe. For particle physics, the knowledge of neutrino masses is important to decide between different models or theories accounting for the non-zero, but very tiny neutrino masses. In order to determine the absolute neutrino mass scale, there are three different approaches:

Cosmology A smearing out of small scale fluctuations of the power spectrum of the universe occurs, depending on the mass of the neutrinos. However, depending on the analysis and data sets, different upper limits on the sum of the three neutrino mass eigenstates can be obtained from cosmological data, e.g. $\sum m\left(v_{i}\right)<0.23 \mathrm{eV} / \mathrm{c}^{2}$ [2] or $\sum m\left(v_{i}\right)<0.98 \mathrm{eV} / \mathrm{c}^{2}$ [2].

Neutrinoless double $\beta$-decay Neutrinoless double $\beta$-decay $(0 v \beta \beta)$ is a forbidden decay in the Standard Model of particle physics, but it could exist in case neutrinos are their own antiparticles (so called Majorana particles). Its discovery would be a direct observation of lepton number violation and could yield information on the neutrino mass via a measurement of the half-life of the decay: $\left(T_{1 / 2}^{0 v \beta \beta}\right)^{-1} \propto m_{e e}^{2}$ with the so called effective neutrino mass $m_{e e}^{2}=\left|\sum U_{e i}^{2} m\left(v_{i}\right)\right|^{2}$. Recent upper limits on $m_{e e}$ are in the range of $0.2-0.4 \mathrm{eV} / \mathrm{c}^{2}$ for the isotopes ${ }^{76} \mathrm{Ge}$ [3] and ${ }^{136} \mathrm{Xe}[4]$.

Direct neutrino mass measurements Direct neutrino mass measurements are based on energy and momentum conservation without further assumptions. One direct method is time of flight measurements of neutrinos using astrophysical sources such as core-collapse supernovae [5]. However, nearby supernovae are rare and the neutrino emission characteristics depend somewhat on the underlying supernova model. Another direct method, which can be investigated with laboratory experiments, is the precision investigation of weak decays such as $\beta$-decay and electron capture. The current upper limit on the neutrino mass of $2 \mathrm{eV} / \mathrm{c}^{2}$ [6] was determined from investigating the tritium $\beta$-spectrum near the endpoint of $18.6 \mathrm{keV}$ by recent experiments in Mainz [7] and Troitsk [8].

\footnotetext{
${ }^{1}$ sometimes also referred to as PMNS matrix
} 


\section{Experiments Investigating $\beta$-Decay}

\section{$2.1 \beta$-Decay}

In nuclear $\beta$-decay a neutron in the atomic nucleus decays into a proton, thereby emitting an electron $\left(e^{-}\right)$and an electron anti-neutrino $\left(\bar{v}_{e}\right)$. The energy released in the decay is stored between the $e^{-}$and $\bar{v}_{e}$ in a statistical way. The energy spectra of the electron is given by the well known Fermi theory of $\beta$-decay [9]:

$$
\frac{d N}{d E} \propto p\left(E+m_{e} c^{2}\right)\left(E_{0}-E\right) \sqrt{\left(E_{0}-E\right)^{2}-m_{\bar{v}_{e}}^{2} c^{4}}
$$

with the the electron energy $E$, the endpoint energy $E_{0}$, the electron mass $m_{e}$ and the average electron anti-neutrino mass $m_{\bar{v}_{e}}^{2}=\sum\left|U_{e i}\right|^{2} m\left(v_{i}\right)^{2}$ which is the incoherent sum of neutrino mass eigenstates and therefore not sensitive to phases of the neutrino mixing matrix (in contrast to neutrinoless double $\beta$-decay). As one can see in equation 2.1, it is the square of the neutrino mass $m_{\bar{v}_{e}}^{2}$ that enters as a parameter. Its effect on the shape of the spectrum is significant only in a very narrow region close to $E_{0}$.

\subsection{The KATRIN Experiment}

(a)

(b)

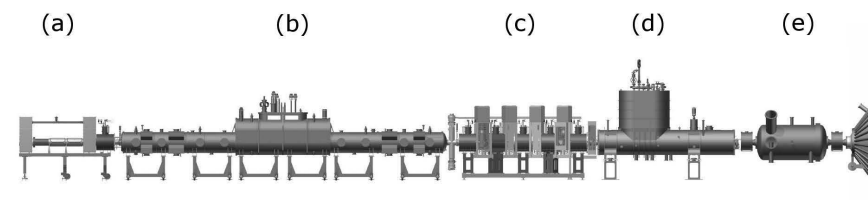

e)

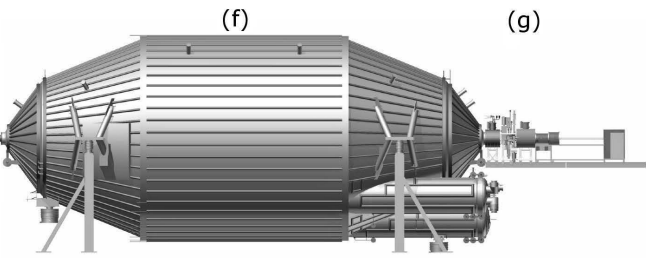

Figure 1: The KATRIN experimental setup with its main components: (a) rear section; (b) windowless gaseous tritium source (WGTS); (c) differential pumping section (DPS); (d) cryogenic pumping section (CPS); (e) pre-spectrometer; (f) main spectrometer; (g) focal plane detector.

The KArlsruhe TRItium Neutrino (KATRIN) experiment [10] is a next generation, large scale experiment to determine the mass of the electron anti-neutrino by investigating the kinematics of tritium beta decay with a sensitivity of $200 \mathrm{meV} / \mathrm{c}^{2}$. The experiment is currently under construction at the Karlsruhe Institute of Technology (KIT) in Germany. The measurement setup (see figure 1) has an overall length of $\approx 70 \mathrm{~m}$. Molecular tritium is injected into the windowless gaseous tritium source (a) where it decays with an activity of $10^{11} \mathrm{~Bq}$, thus providing a sufficient number of $\beta$ decay electrons close to the endpoint energy $E_{0}$. The tritium is removed from the beamline in the differential pumping section (b) and the cryogenic pumping section (c) while electrons from the source are magnetically guided towards the spectrometer section. Both pre-spectrometer and main spectrometer are operated as electrostatic retarding high pass filters of MAC-E filter type [11]. The pre-spectrometer $(\mathbf{d})$ is operated as a pre-filter in order to reduce the flux of electrons into the main spectrometer (e) which performs the energy analysis of the $\beta$-decay electrons near the endpoint with an energy resolution of $\Delta E=0.93 \mathrm{eV}$ at $18.6 \mathrm{keV}$. The main spectrometer is equipped with a dual layer wire electrode system for electrostatically shielding secondary electrons from the inner vessel surface [12]. The transmitted $\beta$-decay electrons are counted in the detector system (f) with 
a segmented silicon detector. Many of the major components of the experiment are already on site (DPS, pre-spectrometer, main spectrometer and detector system) and the remaing components (WGTS, CPS) will be delivered in 2015. The first tritium measurements are expected to start in 2016.

The KATRIN sensitivity of $200 \mathrm{meV} / \mathrm{c}^{2}$ is based on a low background rate of $10^{-2}$ counts per second (cps) and an energy resolution of about $1 \mathrm{eV}$. In order to investigate the initial background performance and electron transmission characteristics of the main spectrometer a series of commissioning measurements was performed in 2013.
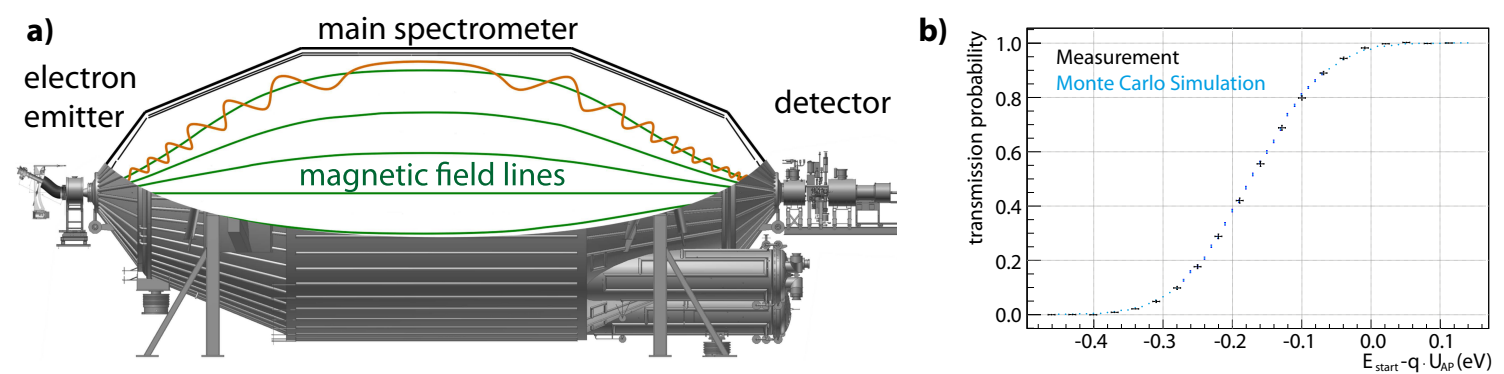

Figure 2: a) Measurement configuration during the first main spectrometer and detector commissioning phase. A photoelectric electron emitter is mounted on the upstream side of the spectrometer, the detector system on the downstream side. b) Simulated and measured transmission function of the main spectrometer. The good agreement shows that the main spectrometer works as expected.

A photoelectric electron emitter (e-gun) was installed on a manipulator at the upstream side of the main spectrometer which allows to shoot electrons of a dedicated energy along a selectable magnetic field line through the spectrometer towards the detector system attached at the downstream side (see Figure 2a)). This configuration was used to measure transmission functions for different retarding potentials of the main spectrometer and manipulator positions (for example see Figure $2 \mathrm{~b}$ ). The width of the transmission functions is dominated by systematic effects of the e-gun (energy and angular spread). The good agreement of simulations, taking into account the energy and angular spread of the e-gun, and the measured data shows that there is no additional broadening caused by the spectrometer. Therefore it is concluded that the main spectrometer works as a MAC-E filter and an energy resolution of about $1 \mathrm{eV}$ seems feasible.

Initial measurements of the main spectrometer background rate, with a not yet background optimized system, resulted in a value of about $1 \mathrm{cps}$. This is a remarkably low initial background rate compared to the commissioning of previous spectrometers such as the KATRIN pre-spectrometer, where Penning discharges caused extreme large backgrounds [13]. A major background source in the KATRIN spectrometers is due to ${ }^{220} \mathrm{Rn}$ and ${ }^{219} \mathrm{Rn}[14,15,16]$. Radon emanates from materials inside the vacuum region of the spectrometer, in particular from the non-evaporable getter (NEG) pump and decays in the volume of the spectrometer. Electrons emitted in processes accompanying the Rn $\alpha$-decay, such as shake-off, internal conversion of excited levels in the Rn daughter atoms, Auger and Coster-Kronig electrons are magnetically trapped inside the spectrometer (see Figure 3a)). Depending on their initial energy, which can be on the order of up to $10 \mathrm{keV}$, the trapped electrons can produce hundreds of secondary electrons via subsequent ionization of residual gas molecules. These secondary electrons are accelerated by the retarding potential as they leave the 

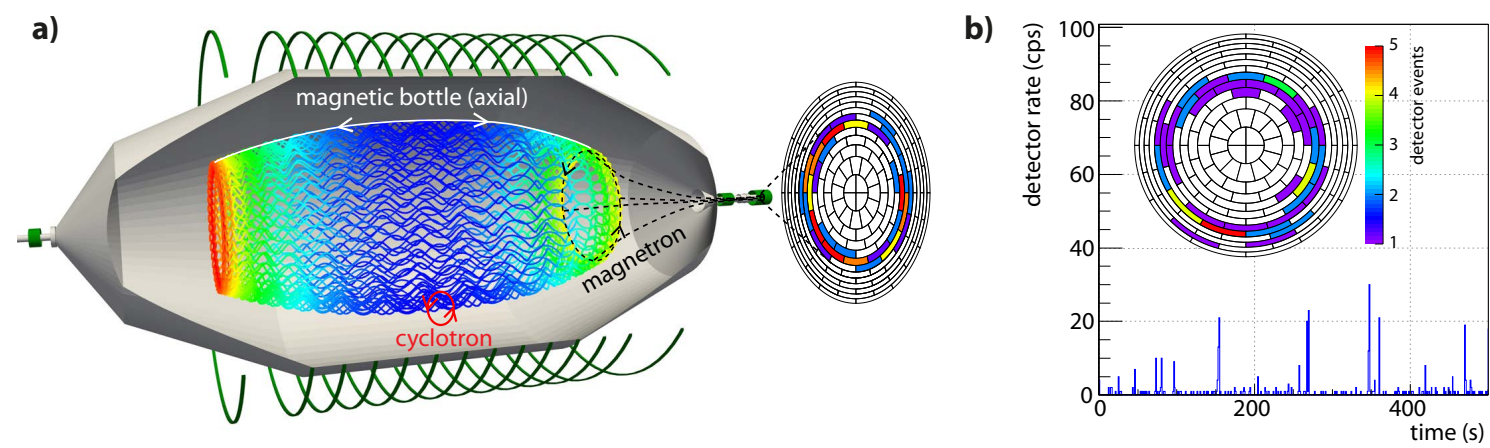

Figure 3: a) Simulation of a magnetically stored electron in the volume of the main spectrometer and pixel distribution at the detector of secondary electrons, produced in ionization processes of the trapped electron with residual gas molecules. b) Background measurement at elevated pressure (about $10^{-8}$ mbar). Each spike in the background rate is initially caused by a radon decay in the volume of the spectrometer. As expected from the simulation, the background events during a spike show a ring-shaped pattern on the detector.

spectrometer and have the same energy as signal electrons from tritium $\beta$-decay by the time they reach the detector. In a measurement with increased pressure (argon, $10^{-8} \mathrm{mbar}$ ), in order to decrease the time between ionizations, the radon induced background is clearly visible as spikes in the background rate with the typical ring-shaped distribution of secondary electrons on the detector (see Figure 3b)). A liquid nitrogen cooled baffle system installed in the pump ports of the main spectrometer, between the NEG pump and the main volume of the spectrometer, could reduce the overall background rate from $1 \mathrm{cps}$ to $0.6 \mathrm{cps}$. The remaining background is assumed to be due to cosmic muon induced secondary electrons from the large inner surface $\left(690 \mathrm{~m}^{2}\right)$ of the spectrometer and radon decays not prevented by the baffle system.

A second main spectrometer and detector commissioning measurement phase has started in October 2014 with the goal to better understand the remaining background sources and to further suppress them with active methods, improved magnetic shielding via a better magnetic field homogeneity and improved electrostatic shielding using the inner wire electrode in dual layer configuration.

\subsection{Project 8}

The Project 8 collaboration proposes to detect coherent cyclotron radiation emitted by energetic electrons in a magnetic field $B$ in order to perform $\beta$-spectroscopy [17] (see Figure 4a)). For mildly relativistic electrons, like those in tritium decay, the relativistic shift of the cyclotron frequency $\omega(\gamma)=\frac{\omega_{0}}{\gamma}=\frac{q B}{m_{e}+E}$ can be used to determine the electron energy $E$ from the emitted radiation.

An experiment using a ${ }^{83 m} \mathrm{Kr}$ source, constructed by the Project 8 collaboration in order to test the feasibility of this approach, was able to detect the coherent synchrotron emission from a single electron for the first time [18]. Figure 4b) shows a typical signal of a $30 \mathrm{keV}$ electron from the decay of ${ }^{83 m} \mathrm{Kr}$. As expected, the frequency increases slowly as the electron loses energy by emission of cyclotron radiation. The sudden jumps result from energy loss caused by collisions with residual gas molecules, predominantly hydrogen. 
a)

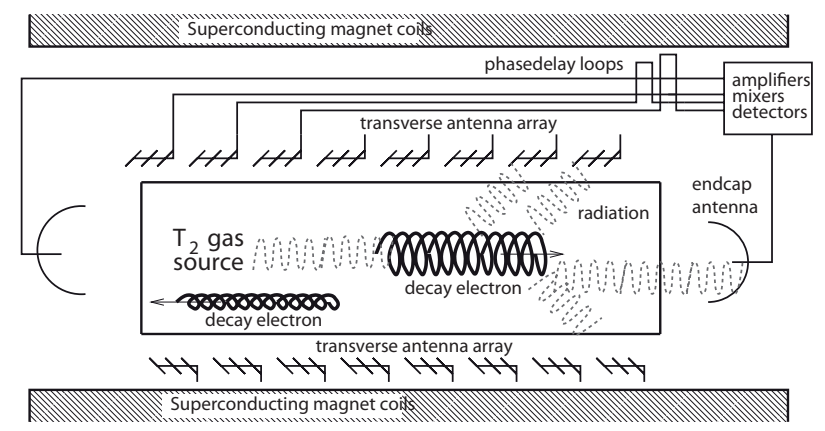

b)

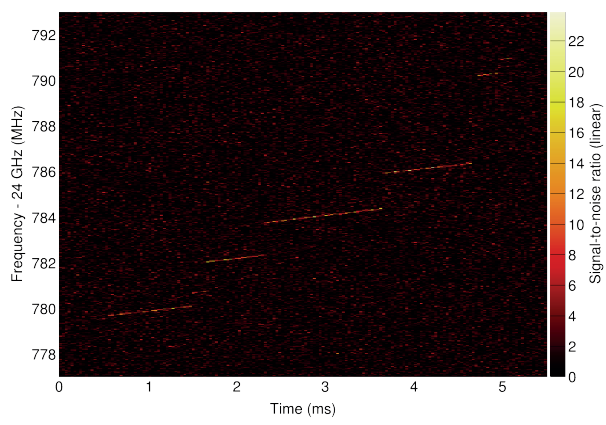

Figure 4: a) Measurement principle: electrons produced from $\beta$-decay undergo cyclotron motion in a magnetic field and emit cyclotron radiation, which is detected by an antenna array. Figure from [17]. b) Typical signal from measurements using a ${ }^{83 m} \mathrm{Kr}$ source, for details see text. Figure from [18].

\section{Calorimetric Experiments}

\subsection{Electron Capture}

Electron capture is a process in which a proton in the atomic nucleus absorbs an inner shell electron, thereby changing into a neutron and simultaneously emitting an electron neutrino. A nonzero neutrino mass affects the de-excitation energy spectrum of the atomic shell after the electron capture.

$$
\frac{d N}{d E} \propto A\left(Q_{E C}-E_{C}\right)^{2} \sqrt{1-\frac{m_{v}^{2}}{\left(Q_{E C}-E_{C}\right)^{2}}} \sum C_{H} n_{H} B_{H} \phi_{H}^{2}(0) \frac{\frac{\Gamma_{H}}{2 \pi}}{\left(E_{C}-E_{H}\right)^{2}+\frac{\Gamma_{H}^{2}}{4}}
$$

A precise calorimetric measurement of this spectrum, including all atomic de-excitation processes such as x-ray emission, Auger electrons and Coster-Kronig transitions, can be used to determine the neutrino mass [19].

\subsection{ECHo}

The ECHo experiment aims to investigate the electron neutrino mass in the sub-eV range using arrays of metallic magnetic calorimeters (MMCs) to calorimetrically measure the energy spectrum following electron capture in ${ }^{163} \mathrm{Ho}$ with high precision and high statistics [21].

A first prototype detector chip consisting of four MMCs has been produced and tested. The measured ${ }^{163} \mathrm{Ho}$ spectrum is shown in Figure 5b). The MMCs energy resolution $\Delta E_{F W H M}=7.6 \mathrm{eV}$ at $6 \mathrm{keV}$ and signal rise-time $\tau_{r} \approx 100 \mathrm{~ns}$ have been measured [21].

Presently the ECHo collaboration is developing and testing new chips consisting of 64 MMCs which are read out using a microwave multiplexing technique [22]. 
a)

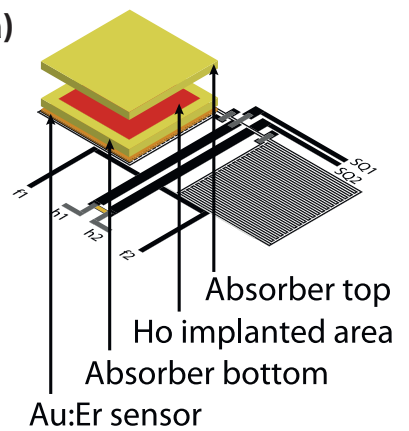

b)

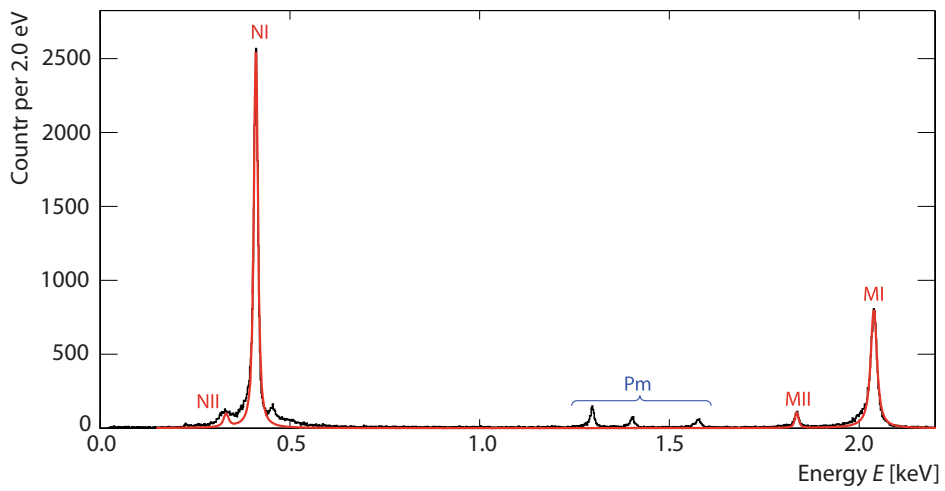

Figure 5: a) Schematic of a metallic magnetic calorimeter (MMC) detector used for ${ }^{163}$ Ho electron capture spectrum measurement. Figure from [20]. b) Measured ${ }^{163}$ Ho spectrum using MMC detectors. The data (black) has been fitted with the theoretical spectrum (red). Figure from [21].

\section{Conclusions}

Direct neutrino mass measurements are a model independent way to determine the neutrino mass. A major improvement of the neutrino mass sensitivity by one order of magnitude is expected of the KATRIN experiment, which is currently under construction and aims to start taking neutrino mass data with tritium in 2016. The Project 8 collaboration was able to successfully detect synchrotron radiation emitted from a single, mildly relativistic electron for the first time, allowing for a new method to perform spectroscopy. This technique has the potential to go beyond the neutrino mass sensitivity reachable with electrostatic spectrometers, in the long-term. An alternative to $\beta$-spectroscopy is the calorimetric measurement of the energy spectrum following electron capture using cryogenic micro-calorimeters. A prototype system consisting of four metallic magnetic calorimeters, operated by the ECHo collaboration, showed a good performance with an energy resolution on the order of $1 \mathrm{eV}$ and signal rise times on the order of $100 \mathrm{~ns}$. If it is feasible to construct an experiment with arrays of ten thousands of calorimeters, this technique could be competitive with KATRIN.

\section{References}

[1] T. Schwetz et al., Global neutrino data and recent reactor fluxes: the status of three-flavour oscillation parameters, New Journal of Physics 13 (2011) http://doi.org/10.1088/1367-2630/13/6/063004

[2] Planck Collaboration, Planck 2013 results. XVI. cosmological parameters, Astronomy and Astrophysics (2014) http://doi.org/10.1051/0004-6361/201321591

[3] GERDA Collaboration, Results on neutrinoless Double- $\beta$ decay of ${ }^{76}$ Ge from phase I of the GERDA experiment, Physical Review Letters 111 (2013) http://doi.org/10.1103/PhysRevLett.111.122503

[4] EXO Collaboration, Search for Majorana neutrinos with the first two years of EXO-200 data, Nature 510 (2014) http://doi.org/10.1038/nature13432

[5] G. Pagliaroli et al., Neutrino mass bound in the standard scenario for supernova electronic antineutrino emission, Astroparticle Physics 33 (2010)

http://doi.org/10.1016/j.astropartphys.2010.02.007 
[6] Particle Data Group, Review of Particle Physics, Chinese Physics C 38 (2014) http://doi.org/10.1088/1674-1137/38/9/090001

[7] C. Kraus et al., Final results from phase II of the Mainz neutrino mass search in tritium $\beta$ decay, The European Physical Journal C 40 (2005) http://doi.org/10.1140/epjc/s2005-02139-7

[8] V.N. Aseev et al., Upper limit on the electron antineutrino mass from the Troitsk experiment, Physical Review D 84 (2011) http://doi.org/10.1103/PhysRevD.84.112003

[9] E. Fermi, Versuch einer Theorie der $\beta$-Strahlen, Zeitschrift für Physik 88 (1934) http://doi.org/10.1007/BF01351864

[10] KATRIN Collaboration, KATRIN design report, FZKA scientific report 7090 (2005) http://bibliothek.fzk.de/zb/berichte/FZKA7090.pdf

[11] G. Beamson et al., The collimating and magnifying properties of a superconducting field photoelectron spectrometer, Journal of Physics E: Scientific Instruments 13 (1980) http://doi.org/10.1088/0022-3735/13/1/018

[12] K. Valerius, The wire electrode system for the KATRIN main spectrometer, Progress in Particle and Nuclear Physics 64 (2010) http://doi.org/10.1016/j.ppnp.2009.12.032

[13] F.M. Fränkle et al., Penning discharge in the KATRIN pre-spectrometer, Journal of Instrumentation 9 (2014) http://doi.org/10.1088/1748-0221/9/07/P07028

[14] F.M. Fränkle et al., Radon induced background processes in the KATRIN pre-spectrometer, Astroparticle Physics 35 (2011) http://doi.org/10.1016/j.astropartphys.2011.06.009

[15] N. Wandkowsky et al., Validation of a model for radon-induced background processes in electrostatic spectrometers, Journal of Physics G: Nuclear and Particle Physics 40 (2013) http://doi.org/10.1088/0954-3899/40/8/085102

[16] S. Mertens et al., Background due to stored electrons following nuclear decays in the KATRIN spectrometers and its impact on the neutrino mass sensitivity, Astroparticle Physics 41 (2013) http://doi.org/10.1016/j.astropartphys.2012.10.005

[17] B. Monreal and J.A. Formaggio, Relativistic cyclotron radiation detection of tritium decay electrons as a new technique for measuring the neutrino mass, Physical Review D 80 (2009) http://doi.org/10.1103/PhysRevD.80.051301

[18] D.M. Asner et al., Single electron detection and spectroscopy via relativistic cyclotron radiation, arXiv (2014) http://arxiv.org/abs/1408.5362

[19] A. De Rújula and M. Lusignoli, Calorimetric measurements of ${ }^{163}$ holmium decay as tools to determine the electron neutrino mass, Physics Letters B 118 (1982) http://doi.org/10.1016/0370-2693(82)90218-0

[20] P.C.O. Ranitzsch et al., Development of Metallic Magnetic Calorimeters for High Precision Measurements of Calorimetric ${ }^{187}$ Re and ${ }^{163}$ Ho Spectra, Journal of Low Temperature Physics 167 (2012) http://doi.org/10.1007/s10909-012-0556-0

[21] L. Gastaldo, The Electron Capture ${ }^{163}$ Ho Experiment ECHo, Journal of Low Temperature Physics 176 (2014) http://doi.org/10.1007/s10909-014-1187-4

[22] J.A.B. Mates et al., Demonstration of a multiplexer of dissipationless superconducting quantum interference devices , Applied Physics Letters 92 (2008) http://doi.org/10.1063/1.2803852 